\title{
ESCALA DE MIEDO A LA EVALUACIÓN NEGATIVA VERSIÓN BREVE (BFNE): PROPIEDADES PSICOMÉTRICAS EN MUESTRAS CLÍNICAS Y DE UNIVERSITARIOS EN MÉXICO
}

\author{
Marcelo Archibaldo Bravo ${ }^{1 *}$, Fabiola González Betanzos ${ }^{1}$, Álvaro Castillo Navarro², Ferran Padrós Blázquez ${ }^{1}$ \\ ${ }^{1}$ Facultad de Psicología. Universidad Michoacana de San Nicolás de Hidalgo (México) \\ ${ }^{2}$ Instituto Mexicano del Seguro Social. Hospital General de Zona No. 83, Morelia
}

Recibido, diciembre 10/2013

Concepto de evaluación, junio 7/2014

Aceptado, julio 29/2014

\begin{abstract}
Referencia: Bravo, M. A., González Betanzos, F., Castillo Navarro, A. \& Padrós Blázquez, F. (2015). Escala de miedo a la evaluación negativa versión breve (BFNE): propiedades psicométricas en muestras clínicas y de universitarios en México. Acta Colombiana de Psicología, 18(1), 69-77. DOI: 10.14718/ACP.2015.18.1.7
\end{abstract}

Resumen

\begin{abstract}
El objetivo del presente trabajo es analizar en muestras clínicas y de universitarios mexicanos, las propiedades psicométricas de la Escala de Miedo a la Evaluación Negativa versión breve (BFNE), así como estudiar su utilidad diagnóstica al establecer un punto de corte que discrimine entre fobia social y trastorno de ansiedad generalizada. Los participantes fueron estudiantes universitarios, sujetos con fobia social y personas con trastorno de ansiedad generalizada. Se llevaron a cabo las siguientes actividades: 1) Proporcionar la media y desviación estándar del BFNE, para cada una de las muestras. 2) Confirmar la estructura interna de la escala. 3) Evaluar la consistencia interna. 4) Analizar la confiabilidad test-retest. 5) Estudiar la validez convergente y divergente. 6) Realizar un análisis de curvas ROC, en donde se establezca un punto de corte que sea capaz de discriminar entre sujetos con fobia social de aquellos que padecen trastorno de ansiedad generalizada. Se concluye que la Escala de Miedo a la Evaluación Negativa versión breve (BFNE) es una medida válida y confiable, capaz de detectar con gran eficacia la presencia de fobia social en las muestras estudiadas.
\end{abstract}

Palabras clave: fobia social, trastorno de ansiedad social, miedo a la evaluación negativa, psicometría, BFNE

\section{BRIEF VERSION OF THE FEAR OF NEGATIVE EVALUATION SCALE (BFNE): PSYCHOMETRIC PROPERTIES IN CLINICAL AND UNIVERSITY STUDENT SAMPLES IN MEXICO}

\begin{abstract}
The aim of the present study is to analyze the psychometric properties of the Brief version of the Fear of Negative Evaluation Scale (BNFE) in clinical and university student samples in Mexico, as well as to assess their diagnostic value by establishing a cut-off score that discriminates between social phobia and generalized anxiety disorder. The sample of participants consisted of university students, subjects with social phobia and people with generalized anxiety disorder. The following activities were carried out: 1) To obtain the BFNE mean score and standard deviation for each sample, 2) To confirm the internal structure of scale. 3) To assess the internal consistency. 4) To analyze the test-retest reliability; 5) To examine the convergent and divergent validity. 6) To establish, by means of ROC analysis, a cut-off score that is able to discriminate between subjects with social phobia and those with generalized anxiety disorder. It is possible to conclude that the Brief version of the Fear of Negative Evaluation Scale (BFNE) is a reliable and valid measure, and it is able to detect effectively the social phobia in the studied samples.

Key words: Social phobia, social anxiety disorder, fear of negative evaluation, psychometrics, BFNE
\end{abstract}

* General Francisco Villa 450, Dr. Miguel Silva. CP. 58120. Morelia, Michoacán (México).Tel. (+52) 443 312 9909 , Ext. 111. archi6886@yahoo.com 


\title{
ESCALA DE MEDO À AVALIAÇÃO NEGATIVA VERSÃO BREVE (BFNE): PROPRIEDADES PSICOMÉTRICAS EM MOSTRAS CLÍNICAS E DE UNIVERSITÁRIOS NO MÉXICO
}

\begin{abstract}
Resumo
O objetivo do presente trabalho é analisar em mostras clínicas e de universitários mexicanos, as propriedades psicométricas da Escala de Medo à Avaliação Negativa versão breve (BFNE), bem como estudar sua utilidade diagnóstica ao estabelecer um ponto de corte que discrimine entre fobia social e transtorno de ansiedade generalizada. Os participantes foram estudantes universitários, sujeitos com fobia social e pessoas com transtorno de ansiedade generalizada. Foram realizadas as seguintes atividades: 1) Proporcionar a média e desvio padrão do BFNE, para cada uma das mostras. 2) Confirmar a estrutura interna da escala. 3) Avaliar a consistência interna. 4) Analisar a confiabilidade teste-reteste. 5) Estudar a validade convergente e divergente. 6) Realizar uma análise de curvas ROC, onde se estabeleça um ponto de corte que seja capaz de discriminar entre sujeitos com fobia social daqueles que padecem de transtorno de ansiedade generalizada. Conclui-se que a Escala de Medo à Avaliação Negativa versão breve (BFNE) é uma medida válida e confiável, capaz de detectar com grande eficácia a presença de fobia social nas mostras estudadas.

Palavras chave: fobia social, transtorno de ansiedade social, medo à avaliação negativa, psicometria, BFNE
\end{abstract}

\section{INTRODUCCIÓN}

La fobia social (también conocida como trastorno de ansiedad social) consiste en un miedo intenso a una o más situaciones sociales, en donde el individuo está expuesto a la probable evaluación de otras personas, temiendo actuar de cierta manera o mostrar síntomas de ansiedad que sean valorados negativamente (American Psychiatric Association, 2013).

Una característica esencial de este trastorno es que los individuos no sufren malestar cuando realizan actividades en privado, sino solamente manifiestan un deterioro en la conducta cuando están siendo observados (Barlow \& Durand, 2001). Al ser observados, su conducta se convierte en algo susceptible de ser evaluado, y debido a que las personas con fobia social presentan un serie de sesgos cognitivos (Baños, Quero \& Botella, 2005; Eastwood et al., 2005; Lundh \& Öst, 2001; Schofielda, Inhoffb \& Coles, 2013; Spector, Pecknold y Libman, 2003; Spokas, Rodebaugh \& Heimberg, 2007), predicen que van a ser evaluados negativamente por las demás personas. Este miedo a la evaluación negativa respecto a la propia ejecución en las situaciones sociales es considerado como el elemento básico y central de la fobia social (Clark, 2001; Johnson \& Anderson, 2014; Van der Molen et al., 2014).

La prevalencia-vida de este trastorno en la población mexicana es de 4.7\% (Medina-Mora et al., 2003). Asimismo, de acuerdo con diversos estudios (Acarturk, Ron de Graaf, van Straten, ten Have \& Cuijpers, 2008; Furmark, 2002; Simon et al., 2002; Stein, Torgrud \& Walker, 2000; Torgrud et al., 2004; Wong, Sarver \& Beidel, 2012), su presencia se relaciona con un deterioro en la calidad de vida, con una baja tasa de apoyo social y con dificultades académicas, laborales y sociales.

Los individuos que padecen fobia social presentan un grado muy alto de comorbilidad. Los trastornos comórbidos más comunes son otros trastornos de ansiedad (principalmente fobia específica, agorafobia y trastorno de pánico), trastornos de abuso de sustancias (básicamente abuso y dependencia del alcohol) y trastornos del estado de ánimo de tipo unipolar (Acarturk et al., 2008; Chartier, Walker $\&$ Stein, 2003). Es importante recalcar que la presencia de comorbilidad está asociada a un grado mayor de deterioro de la calidad de vida (Wong et al, 2012), y que habitualmente la edad de inicio de la fobia social precede a la del trastorno comórbido (Chartier et al., 2003).

Debe destacarse que en México se estima que solamente el $9.1 \%$ de las personas que padecen fobia social buscan algún tipo de tratamiento (Medina-Mora et al., 2003) debido a las características del propio trastorno. Tomando en cuenta todo lo anterior, es de gran utilidad contar con instrumentos adecuados de medición que nos permitan hacer una detección temprana del trastorno.

De esta manera, el objetivo principal del presente trabajo es analizar en muestras clínicas y de universitarios mexicanos las propiedades psicométricas de la Escala de Miedo a la Evaluación Negativa versión breve (BFNE; Leary, 1983), la cual es considerada un instrumento de fácil y rápida aplicación para la detección de la fobia social. Como objetivo secundario nos propusimos estudiar la utilidad diagnóstica del BFNE a través de un análisis de curvas ROC (Características Operativas del Receptor, por sus siglas en inglés), en donde se pretende establecer un punto de corte que sea capaz de discriminar entre suje- 
tos con fobia social de aquellos que padecen trastorno de ansiedad generalizada (uno de los trastornos con los que frecuentemente puede confundirse).

\section{Participantes}

\section{MÉTODO}

Un total de 416 participantes divididos en tres muestras fueron evaluados en la presente investigación. La muestra "A" $(n=316)$ estuvo formada por estudiantes universitarios de la Facultad de Psicología de la Universidad Michoacana de San Nicolás de Hidalgo (UMSNH), con una media de edad de 19.48 años $(\mathrm{DE}=1.83)$, de los cuales un $82.3 \%$ fueron mujeres. La muestra "B" estuvo constituida por pacientes diagnosticados con fobia social $(n=51)$, cuya edad media fue 34.39 años ( $\mathrm{DE}=13.57$ ), y un $52.9 \%$ eran mujeres. Finalmente, la muestra " $\mathrm{C}$ " estuvo compuesta por pacientes con trastorno de ansiedad generalizada ( $\mathrm{n}$ = 49), con una edad media de 40.49 ( $\mathrm{DE}=13.69)$, siendo mujeres el $34.7 \%$ de los participantes. Las muestras clínicas ("B" y "C") fueron recolectadas de acuerdo con los criterios diagnósticos del DSM-IV-TR (American Psychiatric Association, 2000).

\section{Instrumentos}

Escala de Miedo a la Evaluación Negativa versión breve (Brief version of the Fear of Negative Evaluation Scale, $B F N E$ ). Este instrumento fue desarrollado por Leary (1983), y consiste en una versión breve de la Escala de Miedo a la Evaluación Negativa (FNE; Watson \& Friend, 1969), con la cual tiene una correlación de 0.96 (Leary, 1983). Consiste en doce ítems que se responden en una escala de cinco puntos (de 1 a 5), cuatro de los cuales se califican de manera inversa (2, 4, 7 y 10). En el presente estudio fue utilizada la versión española adaptada por Gallego, Botella, Quero, Baños y García-Palacios (2007). En dicha adaptación (llevada a cabo en sujetos con fobia social), se obtuvo una puntuación media en hombres de $41.95(\mathrm{DE}=9.20)$, y en mujeres de 43.45 ( $\mathrm{DE}=9.23)$. A su vez, el BFNE mostró una consistencia interna con valores de alfa de Cronbach de 0.90 , y presentó dos factores (uno conformado con los ítems directos y otro con los inversos).

Escala de Ansiedad ante la Interacción Social (Social Interaction Anxiety Scale, SIAS). Desarrollado por Mattick \& Clarke (1998), es un instrumento que cuenta con 20 ítems que se responden en una escala de cinco puntos (de 0 a 4), tres de los cuales se califican de manera inversa (5, 9 y 11). La consistencia interna con valores de alfa de Cronbach es de .86 a .94 .
Inventario de Ansiedad de Beck (Beck Anxiety Inventory, $B A I)$. Consta de 21 ítems que se responden en una escala de cuatro puntos (de 0 a 3 ). En la presente investigación se utilizó la versión mexicana adaptada por Robles, Varela, Jurado \& Páez (2001), que presentó una consistencia interna con valores de alfa de Cronbach de .83 .

Inventario de Depresión de Beck (Beck Depresion Inventory, $B D I$ ). Se compone de 21 ítems (y un adicional), en cada uno de los cuales se tiene que elegir entre cuatro enunciados que describen el estado de la persona, lo cual viene siendo una escala de cuatro puntos (de 0 a 3 ). Fue empleada la versión mexicana adaptada por Jurado et al (1998), en la cual se obtuvo una consistencia interna con valores de alfa de Cronbach de .87 en población general.

\section{Procedimiento}

Para formar la muestra "A" de participantes, se asistió directamente a once salones de clase de la Facultad de Psicología de la UMNSH, y se le pidió permiso a cada profesor para aplicar una serie de cuestionarios a los estudiantes. La participación fue voluntaria, e implicó responder de manera grupal un formulario de datos socio-demográficos, así como las escalas BFNE, SIAS, BAI y BDI (tiempo aproximado de aplicación: 30 minutos). Dicha aplicación de instrumentos obtuvo la aprobación del Comité de Ética de la Facultad de Psicología de la UMSNH.

Las muestras " $\mathrm{B}$ " $\mathrm{y}$ " $\mathrm{C}$ " fueron conseguidas por un psiquiatra a través de su práctica clínica. El procedimiento consistía en que si los pacientes cumplían con los criterios diagnósticos del DSM-IV-TR (APA, 2000) para fobia social o trastorno de ansiedad generalizada (y no ambos), les era aplicado el BFNE. Cada uno de los participantes dio su consentimiento informado por escrito.

\section{RESULTADOS}

\section{Datos normativos}

La media del BFNE fue de $30.44(\mathrm{DE}=6.62)$ en la muestra de estudiantes, de 52.16 $(\mathrm{DE}=2.71)$ en las personas con fobia social, y de $33.94(\mathrm{DE}=9.37)$ en los sujetos con trastorno de ansiedad generalizada.

\section{Análisis de la estructura factorial}

En distintos estudios se ha reportado que el BFNE presenta dos factores, uno formado por los ítems directos, y otro por los inversos (Carleton, McCreary, Norton \& Asmundson, 2006; Duke, Krishnan, Faith \& Storch, 2006; 
Gallego et al., 2007; Rodebaugh et al., 2004; Tavoli, Melyani, Bakhtiari, Ghaedi \& Montazeri, 2009; Weeks et al., 2005; Woods \& Rodebaugh, 2005). Así que con el objeto de poner a prueba este modelo de dos factores, se realizó un análisis factorial confirmatorio.

Se estudió el ajuste de tres modelos: 1) modelo unifactorial, 2) modelo de dos factores no relacionados y 3) modelo de dos factores relacionados. Los parámetros de los distintos modelos fueron estimados siguiendo el modelo de Máxima Verosimilitud. Puesto que los datos basados en cuestionarios tipo Likert pueden aparecer en distribuciones que no se ajustan a la ley normal, se utilizó el método de distribución ML-Robusto. Se emplearon los siguientes indicadores de bondad de ajuste: $\mathrm{S}-\mathrm{B} \chi^{2}=\chi^{2}$ escalado de Satorra-Bentler (el valor no significativo indica buen ajuste del modelo); $\chi^{2} / \mathrm{gl}=$ el índice anterior dividido por grados de libertad (valores $\leq 5.0$ indica buen ajuste); GFI = LISREL Goodness of Fit Index; CFI = Comparative Fit Index (valores $\geq 0.90$ señalan buen ajuste); $S R M R=$ Standardized Root Mean Square Residual (valores $\leq 0.08$ indican buen ajuste); RMSEA = Root Mean Square Error of Approximation (valores $\leq 0.06$ indican buen ajuste).

Se observó que los valores de $\mathrm{S}-\mathrm{B} \chi^{2}$ en los tres modelos fueron significativos. Sin embargo, los otros índices sugieren un buen ajuste del modelo de dos factores relacionados, y éste resulta mejor en comparación con el modelo unifactorial o el de dos factores no relacionados (véase Tabla 1).

Consistencia interna y descripción de los items

La consistencia interna se estudió utilizando a los participantes de las tres muestras $(n=416)$. Se obtuvo un alfa de Cronbach de .892 para la escala total, de .926 para el factor 1 (ítems directos), y de .660 para el factor 2 (ítems inversos).

La puntuación media para cada ítem osciló entre 2.20 (ítem 1) y 3.75 (ítem 2). Los ítems que puntuaron más alto fueron el 2 y 10 , y los que puntuaron menos fueron el $1 \mathrm{y}$ 8. El rango de correlaciones de cada uno de los ítems con la puntuación total corregida (puntuación total sin tener en cuenta dicho ítem), osciló entre .303 (ítem 4) y .777 (ítem 1). Sin embargo, se observó que si se eliminara cualquiera de los tres ítems (2, 4 y 10), se incrementaría el alfa de Cronbach de la escala total (véase Tabla 2).

Es de destacar que los ítems inversos (los cuales constituyen un factor) fueron los que puntuaron más alto; los que tuvieron menos correlación con la puntuación total fueron corregidos, y los que al eliminarse (tres de ellos), harían que el alfa de Cronbach de la escala total fuera mayor.

\section{Confiabilidad Test-retest}

Para obtener la confiabilidad test-retest, a una parte de la muestra "A" $(\mathrm{n}=188)$ se le volvió a aplicar el BFNE, después de seis semanas de haberse administrado por primera vez. Se obtuvo una correlación de Pearson entre las dos aplicaciones de .813 , con una $\mathrm{p}<.001$.

\section{Validez convergente y divergente}

Utilizando los datos obtenidos en la muestra "A" se realizaron correlaciones de Pearson para el BFNE, con otras medidas de fobia social, ansiedad y depresión. La correlación más alta del BFNE fue con el SIAS $(\mathrm{r}=.632)$, siendo que esta escala también mide el constructo de fobia social. Después siguió la correlación con el BAI (ansiedad) que fue de .418 y con el BDI (depresión), de .393.

Tabla 1.

Índices de bondad de ajuste para los modelos de un factor, dos factores no relacionados y dos factores relacionados sobre la estructura del BFNE, basado en los análisis factoriales confirmatorios EQS

\begin{tabular}{lcccccc}
\hline \multicolumn{1}{c}{ Versión escala } & S-B $\chi^{2}$ & $\chi^{2} / \mathrm{gl}$ & GFI & CFI & SRMR & RMSEA \\
\hline 1 factor & 262.79 & 4.87 & 0.890 & 0.932 & 0.071 & 0.081 \\
2 factores (NR) & 208.03 & 3.85 & 0.925 & 0.948 & 0.155 & 0.070 \\
2 factores (R) & 129.23 & 2.44 & 0.949 & 0.978 & 0.033 & 0.046 \\
\hline
\end{tabular}

Nota: Índices corregidos (método robusto). S-B $\chi^{2}=\chi^{2}$ escalado de Satorra-Bentler; GFI = LISREL goodness of fit index; CFI = Comparative fit index; SRMR $=$ Standardized root mean square residual; RMSEA $=$ Root mean square error of approximation . 


\section{Validez discriminante}

Se realizó un análisis de curvas ROC para evaluar la capacidad de discriminación de la puntuación del BFNE como criterio de decisión para establecer un diagnóstico de fobia social. Si se toma como juicio de validez el diagnóstico del psiquiatra realizado a partir de los criterios del DSM-IV-TR (APA, 2000), se obtienen para las distintas puntuaciones del BFNE diversos indicadores de validez relacionados con su capacidad para diagnosticar la presencia/ausencia de fobia social. Entre estos indicadores, los más utilizados son: sensibilidad (capacidad del BFNE para detectar correctamente pacientes con fobia social, es decir porcentaje de pacientes que tienen una puntuación por arriba de un punto de corte), especificidad (capacidad del BFNE para identificar correctamente pacientes que no presentan fobia social), valor predictivo positivo (probabilidad de que un paciente identificado por el BFNE con fobia social lo presente realmente), y valor predicho negativo (probabilidad de que un paciente identificado por el BFNE sin fobia social en realidad no la presente).
En la tabla 3 se muestra la precisión diagnóstica del BFNE para el rango de puntuaciones totales, que va de 17 a 57 puntos en los pacientes de la muestras "B" $\mathrm{y}$ " $\mathrm{C}$ ". Para evaluar estos resultados es necesario tener en cuenta el contexto clínico e identificar el propósito del test. Si el BFNE se utiliza como instrumento diagnóstico que permita diferenciar entre trastorno de ansiedad social y otro tipo de trastornos, es necesario elegir un punto de corte que ofrezca los máximos valores de sensibilidad y especificidad.

Una forma de analizar ambos indicadores es a través de las curvas ROC. Una curva ROC permite visualizar el rendimiento de las clasificaciones de forma que se representan distintos valores de fracción de falsos positivos (complementario de la especificidad), en el eje de las abscisas, frente a la fracción de verdaderos positivos (sensibilidad) en el eje de ordenadas. Se presenta la gráfica de la curva ROC (véase figura 1) para la puntuación del BFNE. La línea diagonal representa el clasificador aleatorio -diagonal $(0,0)$ y $(1,1)$ - y el clasificador ideal -línea quebrada $(0,0)$ a $(0,1)$ y $(0,1)$ a $(1,1)$. La curva ROC del BFNE se ubica entre

Tabla 2.

Para cada ítem se muestra su media, desviación estándar, correlación ítem-total corregida, y Alfa de Cronbach del total de la escala si dicho ítem es eliminado

\begin{tabular}{ccccc}
\hline Ítem & Media & $\begin{array}{c}\text { Desviación } \\
\text { estándar }\end{array}$ & $\begin{array}{c}\text { Correlación ítem-total } \\
\text { corregida }\end{array}$ & $\begin{array}{c}\text { Alfa de Cronbach si se } \\
\text { elimina el ítem }\end{array}$ \\
\hline 01 & 2.20 & 1.14 & 0.777 & 0.874 \\
$02(\mathrm{I})$ & 3.75 & 1.05 & 0.335 & 0.895 \\
03 & 2.39 & 1.21 & 0.709 & 0.877 \\
$04(\mathrm{I})$ & 3.50 & 1.13 & 0.303 & 0.898 \\
05 & 2.34 & 1.22 & 0.766 & 0.874 \\
06 & 2.39 & 1.35 & 0.724 & 0.876 \\
$07(\mathrm{I})$ & 3.50 & 1.24 & 0.429 & 0.892 \\
08 & 2.23 & 1.20 & 0.698 & 0.878 \\
09 & 2.59 & 1.17 & 0.697 & 0.878 \\
$10(\mathrm{I})$ & 3.51 & 1.19 & 0.405 & 0.893 \\
11 & 2.32 & 1.24 & 0.690 & 0.878 \\
12 & 2.79 & 1.21 & 0.668 & .879 \\
\hline
\end{tabular}

(I): ítem inverso. 
ambos clasificadores extremos, aunque se encuentra más próxima a la situación de clasificación ideal. Asimismo, el área bajo la curva ROC del BFNE indica un rendimiento del clasificador excelente, ya que el área bajo la curva es mayor a 0.97 (AUCBFNE= 0.985), se considera excelente a partir de dicho valor. Esto significa que el $98.5 \%$ de las veces un paciente seleccionado aleatoriamente del grupo con fobia social, tiene una puntuación en el BFNE superior que la que tendría un paciente elegido al azar del grupo sin dicho trastorno. Además, el intervalo de confianza asintótico al 95\% para el área no contiene el valor $0.05(0.96,1.0)$ lo que indica que el BFNE tiene capacidad para distinguir

Tabla 3.

Sensibilidad, especificidad, valores predichos positivos/negativos y proporción correcta total para varias puntuaciones obtenidas en el BFNE (las cuales son definidas como puntos de corte para diagnosticar la presencia del trastorno)

\begin{tabular}{|c|c|c|c|c|c|c|}
\hline $\begin{array}{l}\text { Puntos de } \\
\text { corte }\end{array}$ & $\begin{array}{c}\text { Puntuación } \\
\text { BFNE }\end{array}$ & Sensibilidad & Especificidad & $\begin{array}{l}\text { Valores } \\
\text { predichos } \\
\text { positivos }\end{array}$ & $\begin{array}{c}\text { Valores } \\
\text { predichos } \\
\text { negativos }\end{array}$ & $\begin{array}{l}\text { Proporción } \\
\text { correcta total }\end{array}$ \\
\hline 1 & 17 & 0.515 & 1.000 & 1.000 & 0.020 & 0.520 \\
\hline 2 & 18 & 0.520 & 1.000 & 1.000 & 0.040 & 0.529 \\
\hline 3 & 19 & 0.525 & 1.000 & 1.000 & 0.060 & 0.539 \\
\hline 4 & 22 & 0.536 & 1.000 & 1.000 & 0.100 & 0.559 \\
\hline 5 & 23 & 0.542 & 1.000 & 1.000 & 0.120 & 0.569 \\
\hline 6 & 24 & 0.547 & 1.000 & 1.000 & 0.140 & 0.578 \\
\hline 7 & 26 & 0.559 & 1.000 & 1.000 & 0.180 & 0.598 \\
\hline 8 & 27 & 0.598 & 1.000 & 1.000 & 0.300 & 0.657 \\
\hline 9 & 28 & 0.619 & 1.000 & 1.000 & 0.360 & 0.686 \\
\hline 10 & 29 & 0.634 & 1.000 & 1.000 & 0.400 & 0.706 \\
\hline 11 & 32 & 0.650 & 1.000 & 1.000 & 0.440 & 0.725 \\
\hline 12 & 33 & 0.658 & 1.000 & 1.000 & 0.460 & 0.735 \\
\hline 13 & 34 & 0.675 & 1.000 & 1.000 & 0.500 & 0.755 \\
\hline 14 & 36 & 0.684 & 1.000 & 1.000 & 0.520 & 0.765 \\
\hline 15 & 37 & 0.712 & 1.000 & 1.000 & 0.580 & 0.794 \\
\hline 16 & 38 & 0.722 & 1.000 & 1.000 & 0.600 & 0.804 \\
\hline 17 & 39 & 0.732 & 1.000 & 1.000 & 0.620 & 0.814 \\
\hline 18 & 40 & 0.776 & 1.000 & 1.000 & 0.700 & 0.853 \\
\hline 19 & 42 & 0.800 & 1.000 & 1.000 & 0.740 & 0.873 \\
\hline 20 & 43 & 0.813 & 1.000 & 1.000 & 0.760 & 0.882 \\
\hline 22 & 44 & 0.850 & 0.976 & 0.981 & 0.820 & 0.902 \\
\hline 23 & 45 & 0.864 & 0.977 & 0.981 & 0.840 & 0.912 \\
\hline 25 & 46 & 0.895 & 0.978 & 0.981 & 0.880 & 0.931 \\
\hline 26 & 47 & 0.891 & 0.936 & 0.942 & 0.880 & 0.912 \\
\hline 27 & 48 & 0.941 & 0.922 & 0.923 & 0.940 & 0.931 \\
\hline 28 & 49 & 0.938 & 0.870 & 0.865 & 0.940 & 0.902 \\
\hline 30 & 50 & 1.000 & 0.877 & 0.865 & 1.000 & 0.931 \\
\hline 31 & 51 & 1.000 & 0.820 & 0.788 & 1.000 & 0.892 \\
\hline 32 & 52 & 1.000 & 0.725 & 0.635 & 1.000 & 0.814 \\
\hline 33 & 53 & 1.000 & 0.641 & 0.462 & 1.000 & 0.725 \\
\hline 34 & 54 & 1.000 & 0.562 & 0.250 & 1.000 & 0.618 \\
\hline 35 & 55 & 1.000 & 0.549 & 0.212 & 1.000 & 0.598 \\
\hline 36 & 56 & 1.000 & 0.515 & 0.096 & 1.000 & 0.539 \\
\hline 37 & 57 & 1.000 & 0.500 & 0.038 & 1.000 & 0.510 \\
\hline
\end{tabular}


entre pacientes con fobia social, de los que no lo tienen de manera no aleatoria.

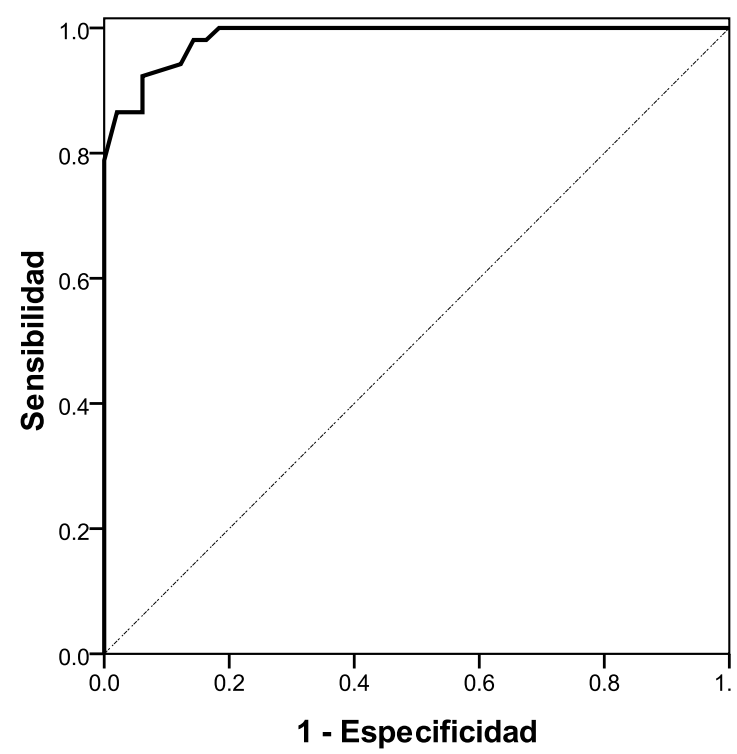

Figura 1. Área bajo la curva ROC para el BFNE. En el gráfico los segmentos diagonales son producidos por los empates

El criterio de decisión óptimo para la clasificación de los pacientes a partir del BFNE depende de diversos factores, como son gravedad de la condición de interés, la prevalencia de dicha condición en la población, la disponibilidad de medidas correctivas para los individuos clasificados y el coste financiero, emocional, etc. que conllevan las falsas alarmas (Adams \& Hand, 1999). A partir de los valores observados de especificidad y sensibilidad, para el BFNE el punto de corte óptimo es de 48.

\section{DISCUSIÓN}

El presente trabajo tuvo como objetivo principal analizar en muestras clínicas y de universitarios mexicanos las propiedades psicométricas de la Escala de Miedo a la Evaluación Negativa versión breve (BFNE; Leary, 1983), la cual, como su nombre sugiere, evalúa la intensidad del miedo ante la posibilidad de ser juzgado negativamente por otras personas (componente básico y central de la fobia social).

Los análisis factoriales confirmatorios llevados a cabo mostraron una estructura bifactorial del BFNE, en la que un factor está compuesto por los ítems directos, y el otro por los inversos. Dicha estructura concuerda con lo reportado en otros estudios (Carleton et al., 2006; Duke et al., 2006; Gallego et al., 2007; Rodebaugh et al., 2004; Tavoli et al., 2009; Weeks et al., 2005; Woods \& Rodebaugh, 2005).
Sin embargo, esta estructura de dos factores ha recibido algunas críticas, ya que se sugiere que en realidad los ítems inversos no conforman un factor, sino que la redacción de dichos ítems no es clara y confunde a los participantes (Gallego et al., 2007; Tavoli et al., 2009; Rodebaugh et al., 2004; Woods \& Rodebaugh, 2005). Ocasiona, de esta manera, un factor que no es teóricamente posible, ya que se supone que todos los ítems (tanto directos como inversos) están midiendo un mismo constructo (miedo a la evaluación negativa) que carece de componentes identificados (Carleton et al., 2006).

Por lo tanto, algunos autores (Gallego, 2010; Rodebaugh et al., 2004) sugieren eliminar los ítems inversos (y sólo tomar en cuenta los directos), por lo cual se ha propuesto utilizar el BFNE-S (Brief version of the Fear of Negative Evaluation scale-Straightforward items). Sin embargo, otros investigadores (Carleton et al, 2006; Collins, Westra, Dozois \& Stewart., 2005) proponen volver a redactar los ítems inversos en forma directa, creando así al BFNE-II (Brief version of the Fear of Negative Evaluation scale-Revised). Sin embargo, como dice Gallego (2010), son necesarios más estudios para saber cuál de estas dos propuestas tiene mejores propiedades psicométricas.

Con respecto a la consistencia interna del BFNE, ésta fue muy buena tanto en el total de la escala como en el factor formado por ítems directos; no obstante, se encontró un alfa más bajo en el factor compuesto por ítems inversos. Estos resultados concuerdan con lo reportado en otras investigaciones (Duke et al., 2006; Gallego et al., 2007; Rodebaugh et al., 2004; Weeks et al., 2005).

Debe destacarse que los ítems inversos tuvieron menor correlación con la puntuación total corregida, y los que al eliminarse (tres de ellos), harían que el alfa de Cronbach de la escala total fuera mayor. Esto, junto a la baja consistencia interna que mostró el factor compuesto por ítems inversos, confirman la pertinencia de realizar alguna modificación respecto a los ítems inversos, ya sea eliminarlos (BFNE-S) o redactarlos en forma directa (BFNE-II).

En lo que concierne a la estabilidad temporal del instrumento, el BFNE mostró una buena confiabilidad test-retest en la muestra de estudiantes, en un período de seis semanas, lo cual coincide con el trabajo de Tavoli et al. (2009), en donde también se obtuvo una buena confiabilidad en estudiantes evaluada en un lapso de tres semanas.

Para analizar la validez convergente y divergente del instrumento se realizaron correlaciones de Pearson entre el BFNE y otras medidas de fobia social, ansiedad y depresión. Los resultados obtenidos fueron congruentes con lo esperado teóricamente, ya que la correlación más alta fue con la escala SIAS que mide fobia social. Después siguieron correlaciones moderadamente elevadas con el 
BAI que evalúa ansiedad en general y el BDI que mide depresión. En otros estudios, el BFNE también ha mostrado correlaciones en el sentido esperado, con el SIAS (Tavoli et al., 2009; Weeks et al., 2005) y con el BDI (Duke et al., 2006; Gallego et al., 2007; Weeks et al., 2005).

El BFNE mostró una gran utilidad diagnóstica, ya que de acuerdo con el análisis de curvas ROC, al tomar en cuenta un punto de corte de 48, el instrumento es capaz de discriminar entre pacientes que padecen fobia social, de aquellos diagnosticados con trastorno de ansiedad generalizada, en el $98.5 \%$ de las ocasiones.

Deben señalarse algunas de las limitaciones de nuestra investigación. En primer lugar, la muestra de estudiantes universitarios estuvo formada en su mayoría por participantes de sexo femenino, por lo que hubiera sido conveniente tener una muestra equilibrada en género. También sería deseable contar con una muestra de población general, para así tener personas de todas las edades y con diferentes niveles de escolaridad.

Respecto a las muestras clínicas, para su recolección se utilizó un procedimiento de tipo incidental, y no se utilizaron procedimientos más adecuados, como muestreos aleatorios o de selección sistemática de todos los pacientes que acudieron a tratamiento. Además, aunque se exigió el uso de criterios DSM-IV-TR, el diagnóstico se realizó a través del juicio clínico de un solo profesional. Hubiera sido deseable la utilización de alguna entrevista estructurada diagnóstica, o haber contado con el juicio diagnóstico e independiente de dos o más clínicos.

Por otro lado, uno de los instrumentos utilizados en la presente investigación para evaluar la presencia de fobia social (Escala de Ansiedad ante la Interacción Social, SIAS; Mattick \& Clarke, 1998), carece de datos psicométricos en población mexicana. En la actualidad (hasta donde nos ha sido posible investigar), no existe ningún instrumento para medir la presencia de fobia social o variables afines adaptado en población mexicana.

Asimismo, sería interesante evaluar la sensibilidad de la escala al cambio después de un tratamiento. Del mismo modo, es necesario en próximos estudios analizar las propiedades psicométricas del BFNE-S y del BFNE-II, para así aportar evidencia sobre la mejor manera de hacer frente a los ítems inversos del BFNE.

En conclusión, podemos señalar que después de estudiar las propiedades psicométricas de la Escala de Miedo a la Evaluación Negativa versión breve (BFNE; Leary, 1983), ésta demostró poseer una adecuada validez y confiabilidad en las muestras estudiadas. Consideramos que debido a la sencillez y brevedad con que puede ser administrada, y también a la elevada eficacia mostrada para detectar la presencia de fobia social, puede ser un instrumento de gran utilidad en la investigación y en el ámbito clínico aplicado.

\section{REFERENCIAS}

Acarturk, C., Ron de Graaf, van Straten, A., Ten Have, M. \& Cuijpers, P. (2008). Social Phobia and Number of Social Fears, and their Association with Comorbidity, Health-Related Quality of Life and Help Seeking. Social Psychiatry and Psychiatric Epidemiology, 43, 273-279.

Adams, N.M., \& Hand, D.J. (1999). Comparing classifiers when the misallocations cost are uncertain. Pattern Recognition, 32, 1139-1147.

American Psychiatric Association (2000). Diagnostic and Statistical Manual of Mental Disorders (4a. ed. texto revisado, DSM-IV-TR). Washington, DC: Autor.

American Psychiatric Association. (2013). Diagnostic and Statistical Manual of Mental Disorders (5th ed.). Arlington, VA: American Psychiatric Publishing.

Baños, R. M., Quero, S., \& Botella, C. (2005). Sesgos atencionales en la fobia social medidos mediante dos formatos de la tarea Stroop emocional (de tarjetas y computerizado) y papel mediador de distintas variables clínicas. Internacional Journal of Clinical and Health Psychology, 5(1), 23-42.

Barlow, D. H. \& Durand V. M. (2001). Evaluación clínica y diagnóstico. En D. H. Barlow y V. M. Durand, Psicología Anormal: un enfoque integral (p. 72-101). México: Thomson.

Carleton, R.N., McCreary, D.R, Norton, P.J. \& Asmundson J.G. (2006). Brief Fear of Negative Evaluation Scale-Revised. Depression and Anxiety, 23, 297-303.

Chartier, M. J., Walker, J. R. \& Stein, M. B. (2003). Considering Comorbidity in Social Phobia. Social Psychiatry \& Psychiatric Epidemiology, 38, 728-734.

Clark, D. M. (2001). A Cognitive Perspective on Social Phobia. En W.R. Crozier y L.E. Alden (Eds.), International Handbook of Social Anxiety: Concepts, Research and Interventions Relating to the Self and Shyness (p. 405-430). London: John Wiley \& Sons.

Collins, K.A., Westra, H.A., Dozois, D. \& Stewart, S.H. (2005). The Validity of the Brief Version of the Fear of Negative Evaluation Scale. Anxiety Disorders, 19, 345-359.

Duke, D., Krishnan, M., Faith, M. \& Storch, E.A. (2006). The Psychometric Properties of the Brief Fear of Negative Evaluation Scale. Journal of Anxiety Disorders, 20(6), 807-817.

Eastwood, J. D., Smilek, D., Oakman, J. M., Farvolden, P., van Ameringen, M., Mancini C., \& Merikle, P. M. (2005). Individuals with Social Phobia are Biased to Become Aware of Negative Faces. Visual Cognition, 12(1), 159-179.

Furmark, T. (2002). Social phobia: overview of community surveys. Acta Psychiatrica Scandinavica, 105, 84-93. 
Gallego, M.J. (2010). Brief Version of the Fear of Negative Evaluation Scale - Straightforward Items (BFNE-S): Psychometric properties in a Spanish population. The Spanish Journal of Psychology, 13(2), 981-989.

Gallego, M. J., Botella, C., Quero, S., Baños, R. \& GarcíaPalacios, A. (2007). Propiedades psicométricas de la escala de miedo a la evaluación negativa versión breve (BFNE) en muestra clínica. Revista de Psicopatología y Psicología Clínica, 12(3), 163-176.

Johnson, S. B. \& Anderson, P. L. (2014). Stereotype Confirmation Concern and Fear of Negative Evaluation Among African Americans and Caucasians with Social Anxiety Disorder. Journal of Anxiety Disorders, 28, 390393.

Jurado, S., Villegas, M. E., Méndez, L., Rodríguez, F., Loperena, V. \& Varela, R. (1998). La estandarización del Inventario de Depresión de Beck para los residentes de la Ciudad de México. Salud Mental, 21(3), 26-31.

Leary, M. R. (1983). A Brief Version of the Fear of Negative Evaluation Scale. Personality and Social Psychology Bulletin, 9, 371-375.

Lundh, L. G. \& Öst, L. G. (2001). Attentional bias, selfconsciousness and perfectionism in social phobia before and after cognitive-behaviour therapy. Scandinavian Journal of Behaviour Therapy, 30(1), 4-16.

Mattick, R. P. \& Clarke, J. C. (1998). Development and validation of measures of social phobia scrutiny fears and social interaction anxiety. Behaviour Research and Therapy, 36, 455-470.

Medina-Mora, M. E., Borges, G., Lara, C., Benjet, C., Blanco, J., Fleiz, C., Villatoro, J., Rojas, E., Zambrano, J., Casanova, L. \& Aguilar-Gaxiola, S. (2003). Prevalencia de trastornos mentales y uso de servicios: Resultados de la encuesta nacional de epidemiología psiquiátrica en México. Salud Mental, 26(4), 1-16.

Robles, R., Varela, R., Jurado, S. \& Páez (2001). Versión mexicana del Inventario de Ansiedad de Beck: Propiedades psicométricas. Revista Mexicana de Psicología, 18(2), 211218.

Rodebaugh, T.L., Woods, C.M., Thissen, D., Heimberg, R.G., Chambles, D.L. \& Rapee, R.M. (2004). More information from fewer questions: The factor structure and item properties of the original and Brief Fear of Negative Evaluation scale. Psychological Assessment, 16(2), 169181 .
Simon, N. M., Otto, M. W., Korbly, N. B., Peters, P. M., Nicolaou, D. C. \& Pollack, M. H. (2002). Quality of life in social anxiety disorder compared with panic disorder and the general population. Psychiatric Services, 53(6), 714-718.

Schofield, C. A., Inhoff, A. W. \& Coles, M. E. (2013). Timecourse of attention biases in social phobia. Journal of Anxiety Disorders, 27, 661-669.

Spector, I. P., Pecknold, J. C. \& Libman, E. (2003). Selective attentional bias related to the noticeability aspect of anxiety symptoms in generalized social phobia. Journal of Anxiety Disorders, 17(5), 517-531.

Spokas, M. E., Rodebaugh, T. L. \& Heimberg, R. G. (2007). Cognitive biases in social phobia. Psychiatry, 6(5), 204210.

Stein, M. B., Torgrud, L. J. \& Walker, J. R. (2000). Social phobia symptoms, subtypes, and severity: Findings from a community survey. Arch Gen Psychiatry, 57, 1046-1052.

Tavoli, A., Melyani, M., Bakhtiari, M., Ghaedi, G. \& Montazeri, A. (2009). The Brief Fear of Negative Evaluation scale (BFNE): Translation and validation study of the Iranian version. BMC Psychiatry, 9(42).

Torgrud, L. J., Walker, J. R., Murray, L., Cox, B. J., Chartier, M. \& Kjernisted, K. D. (2004). Deficits in perceived social support associated with generalized social phobia. Cognitive Behaviour Therapy, 33(2), 87-96.

Van der Molen, M. J. W., Poppelaars, E. S., Van Hartingsveldt, C., Harrewijn A., Moor, B. G. \& Westenberg, M. (2014). Fear of negative evaluation modulates electrocortical and behavioral responses when anticipating social evaluative feedback. Frontier in Human Neuroscience, 7, article 936.

Watson, D. \& Friend, R. (1969). Measurement of socialevaluative anxiety. Journal of Consulting and Clinical Psychology, 33, 448-457.

Weeks, J. W., Heimberg, R.G., Fresco, D. M., Hart, T. A., Turk, C. L., Schneier, F. R. \& Liebowitz, M. R. (2005). Empirical validation and psychometric evaluation of the Brief Fear of Negative Evaluation scale in patients with social anxiety disorder. Psychological Assessment, 17(2), 179-190.

Wong, N., Sarver, D. E. \& Beidel, D. C. (2012). Quality of life impairments among adults with social phobia: The impact of subtype. Journal of Anxiety Disorders, 26, 50-57.

Woods, C.M. \& Rodebaugh, T.L. (2005). Factor structures of the original and Brief Fear of Negative Evaluation (FNE and BFNE) scales: Correction to an erroneous footnote. Psychological Assessment, 17(3), 385-386. 